\title{
MODELAGEM MATEMÁTICA NA LICENCIATURA EM MATEMÁTICA: RELATO DE UMA EXPERIÊNCIA
}

\author{
Edilaine Regina dos Santos ${ }^{1}$ \\ Brunna Leonardi Caciolato ${ }^{2}$ \\ Alisson Henrique dos Santos ${ }^{3}$ \\ Fernanda Felix Silva ${ }^{4}$
}

\begin{abstract}
Resumo
Esse artigo relata uma experiência com um grupo de alunos da licenciatura em Matemática de uma universidade pública paranaense no desenvolvimento de uma modelagem matemática em uma disciplina desse curso. Após uma familiarização com tarefas de modelagem matemática, os alunos se envolveram em processo de obtenção de um modelo matemático para o estudo da taxa de alfabetização da população brasileira com 15 anos ou mais. Por meio desse trabalho, e do contato com diferentes tarefas, os futuros professores puderam vivenciar a modelagem matemática em sala de aula e ter ideias de como poderão lidar com essa forma de condução da aula de Matemática.
\end{abstract}

Palavras-chave: Educação Matemática, Modelagem Matemática, Modelo Matemático.

\section{MATHEMATICAL MODELING IN THE PRESERVICE MATHEMATICS TEACHERS EDUCATION: AN EXPERIENCE REPORT}

\begin{abstract}
This paper presents an experience with students from a Mathematics course of a public university of Paraná in the development of a mathematical modeling. The prospective Mathematics teachers engaged, after a familiarization with mathematical modeling tasks, in a process of obtaining a mathematical model for literacy rate of the Brazilian population age 15 and above. Through this work, and performing different tasks, prospective teachers were able to experience mathematical modeling in the classroom and to think about how they can handle this instructional approach in Mathematics classes.
\end{abstract}

Keywords: Mathematics Education. Mathematical Modeling. Mathematical Model.

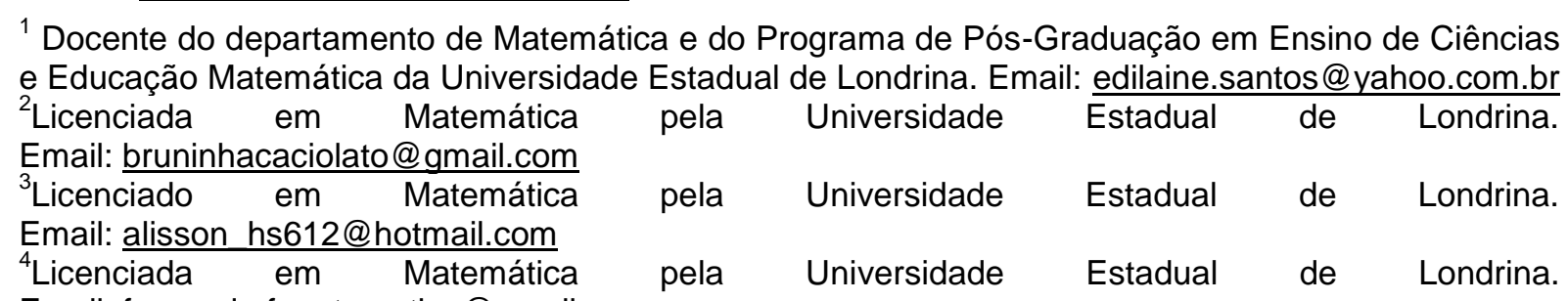
Email: fernanda.f.matematica@gmail.com

Criar Educação, Criciúma, v. 7, nº1, jan/jul 2018.- PPGE - UNESC 


\section{Introdução}

Esse artigo relata uma experiência com um grupo de alunos da licenciatura em Matemática de uma universidade pública paranaense no desenvolvimento de um trabalho na disciplina Modelagem Matemática na perspectiva da Educação Matemática no ano letivo de 2016.

Nessa disciplina, os alunos tiveram a possibilidade de, entre outras coisas, conhecer a modelagem matemática como uma possibilidade para a condução das aulas de Matemática, estudar e analisar modelos matemáticos clássicos e o conteúdo matemático correspondente, e elaborar modelos alternativos. Para isso, dentre outras ações, desenvolveram trabalhos com tarefas de modelagem matemática, inseridas nas aulas de acordo com diferentes momentos de familiarização.

No caso do relato em tela, o trabalho ocorreu mediante um processo de modelagem conduzido pelos alunos ${ }^{5}$, assessorado pela professora ${ }^{6}$ responsável pela disciplina nesse ano letivo, em que elaboraram um modelo matemático para o estudo da taxa de alfabetização da população brasileira com 15 anos ou mais.

A seguir são apresentadas algumas considerações sobre a modelagem matemática, na perspectiva adotada na disciplina ofertada aos alunos, o relato da elaboração desse modelo e algumas considerações sobre essa experiência.

\section{Modelagem Matemática no âmbito da Educação Matemática: algumas considerações}

De um modo geral, a modelagem matemática pode ser entendida como "um processo dinâmico utilizado para a obtenção e validação de modelos matemáticos", consistindo "[...] na arte de transformar situações da realidade em problemas matemáticos cujas soluções devem ser interpretadas na linguagem usual" (BASSANEZI, 2011, p.24).

\footnotetext{
${ }^{5}$ Segundo, terceiro e quarto autores desse artigo.

${ }^{6}$ Primeira autora desse artigo.
}

Criar Educação, Criciúma, v. 7, ำ1, jan/jul 2018.- PPGE - UNESC 
No contexto educacional ela tem sido indicada como uma das tendências em

Educação Matemática pelo qual o professor pode fundamentar sua prática docente para o trabalho com os alunos acerca dos conteúdos matemáticos (PARANÁ, 2008) e o aluno, a vir a se interessar pelo estudo da Matemática (PEREIRA et al., 2017). Nesse sentido, o "fenômeno modelado deve servir de pano de fundo ou motivação para o aprendizado das técnicas e conteúdos da própria matemática" (BASSANEZI, 2011, p.38).

Para isso, o trabalho dos alunos segundo essa tendência pode ser pautado por algumas ações, tais como "identificação e seleção de variáveis, a elaboração de hipóteses, a obtenção de um modelo matemático, a resolução do problema por meio de procedimentos adequados e a análise da solução, identificando a sua aceitabilidade ou não" (ALMEIDA, 2006, p.122), e ocorrer gradativamente mediante a familiarização desses com tarefas desse tipo nas aulas de Matemática. Essa familiarização, segundo Almeida e Dias (2004, p.25), pode ocorrer respeitando diferentes momentos, tais como:

- Em um primeiro momento, são abordadas, com todos os alunos, situações em que estão em estudo a dedução, a análise e a utilização de um modelo matemático, a partir de uma situação problema já estabelecida e apresentada pelo professor; neste momento, a formulação de hipóteses e a investigação do problema, que resulta na dedução do modelo, são realizadas em conjunto com todos os alunos e o professor;

- Posteriormente, uma situação problema já reconhecida, juntamente com um conjunto de informações, pode ser sugerida pelo professor à classe, e os alunos, divididos em grupos, realizam a formulação das hipóteses simplificadoras e a dedução do modelo durante a investigação e, a seguir, validam o modelo encontrado;

- Finalmente, os alunos, distribuídos em grupos, são incentivados a conduzirem um processo de Modelagem, a partir de um problema escolhido por eles, devidamente assessorados pelo professor.

Entretanto, cabe salientar que essa proposta de familiarização dos alunos com tarefas de modelagem matemática, bem como qualquer outra, não deve ser entendida como algo rígido, tendo em vista que, conforme destaca Barbosa ${ }^{7}$ (2009, p.6),

[...] a escolha sobre a forma de inserir atividades de Modelagem na escola depende das oportunidades e limitações do contexto escolar, da maneira que o professor entende sua função de ensinar e o perfil dos alunos. Muitas

7 O autor utiliza a palavra "casos" para se referir as formas de organização do trabalho com modelagem matemática em sala de aula. Nesse artigo, consideramos "casos" e "momentos" como sinônimos.

Criar Educação, Criciúma, v. 7, nº1, jan/jul 2018.- PPGE - UNESC 
vezes, não é possível implementarmos o caso 3; então, talvez, possamos desenvolver o 1, avaliar o processo e, então, dar outro passo. Outras vezes, podemos implementar o caso 3 diretamente. Enfim, isso depende do contexto escolar e da decisão do professor.

O trabalho com os alunos relatado nesse artigo foi desenvolvido segundo a perspectiva do terceiro momento, conforme descrito por Almeida e Dias (2004), e foi pautado pelas seguintes ações: escolha do tema, seleção de dados, definição do problema, definição de variáveis, definição de hipóteses, obtenção do modelo, validação do modelo, interpretação dos resultados e resolução do problema.

\section{Relatando a experiência}

$\mathrm{Na}$ disciplina, conforme mencionado anteriormente, os alunos também trabalharam com tarefas de modelagem matemática inseridas nas aulas de acordo com diferentes momentos de familiarização. No ano de 2016, durante aulas dos três primeiros bimestres, os alunos puderam trabalhar com as seguintes tarefas:

Quadro 1: tarefas trabalhadas na disciplina no ano de 2016

\begin{tabular}{|c|c|}
\hline Título da tarefa & Fonte \\
\hline Numeração do calçado & adaptado de Brito (2004) \\
\hline Ideb do Ensino Médio & adaptado de Costa e Silva (2014) \\
\hline Álcool e direção & adaptado de Oliveira et al (2014) \\
\hline Efeito estufa & adaptado de Gerolômo e Vertuan (2009) \\
\hline Ritalina & adaptado de Pfahl et al (2014) \\
\hline Decaimento radioativo & adaptado de Silva e Vertuan (2009) \\
\hline Aquecimento global & adaptado de Ferreira et al (2009) \\
\hline
\end{tabular}

Fonte: notas de aula da disciplina

As tarefas Numeração do calçado, Ideb do Ensino Médio, Álcool e direção, e Efeito estufa foram conduzidas em sala de aula de acordo com o primeiro momento de familiarização dos alunos com tarefas de modelagem matemática, conforme Almeida e Dias (2004). Já as tarefas Ritalina, Decaimento radioativo e Aquecimento global foram realizadas com os alunos sendo esses responsáveis pela formulação

Criar Educação, Criciúma, v. 7, ำ1, jan/jul 2018.- PPGE - UNESC 


\section{(190)}
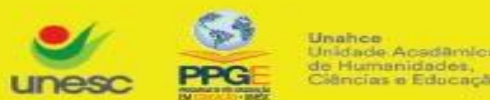

das hipóteses, dedução do modelo e validação desse, além da intepretação dos resultados e resolução do problema, ou seja, de acordo com o segundo momento.

Mediante o trabalho com esses materiais os alunos tiveram a oportunidade de realizar algumas ações em um processo de familiarização com modelagem matemática e, além disso, estudar o conteúdo matemático presente no desenvolvimentos dos modelos matemáticos.

Após isso, eles foram orientados quanto ao desenvolvimento de uma modelagem matemática, em grupo, em que seriam responsáveis pela condução de todo o trabalho, ou seja, desde a escolha do tema até a resolução do problema. Para isso, foram disponibilizadas duas aulas semanais de 50 minutos no 4ํㅡㄹ bimestre do ano letivo de 2016, totalizando 10 aulas. Nesse período, a cada semana, os alunos foram assessorados pela professora da disciplina.

A seguir é apresentado o relato de um trabalho relacionado ao tema "taxa de alfabetização da população brasileira de 15 anos ou mais", desenvolvido por um grupo de alunos.

O trabalho teve início com a busca por temas e dados que pudessem dar origem a um problema e propiciar algum estudo. Em princípio os alunos buscaram por temas que lhes interessavam, mas perceberam que por algumas vezes os dados não eram suficientes para a realização de uma modelagem e por outras, não podiam assegurar a confiabilidade das fontes que os disponibilizavam. Frente à isso, mudaram a estratégia: optaram por buscar dados que pudessem ser manipulados visando a obtenção de um modelo matemático e que fossem de algum tema atual e relevante. Desse modo, mediante o acesso a alguns sites, encontraram estatísticas referentes a taxa de alfabetização da população brasileira de 15 anos ou mais e sua meta para 2024. A seguir são apresentadas algumas informações referentes ao tema e os dados obtidos para o desenvolvimento da modelagem matemática. 
Quadro 2: informações a respeito do tema e dos dados

\begin{abstract}
A taxa de alfabetização é um indicador "calculado com base nos dados da Pesquisa Nacional por Amostra de Domicílios (Pnad), que pergunta aos entrevistados maiores de 15 anos se sabem ler e escrever. A taxa de alfabetização, portanto, considera alfabetizadas as pessoas que declaram saber ler e escrever"8.

A figura a seguir apresenta informações para a taxa de alfabetização da população de 15 ou mais e uma estimativa dessa para 2024, que leva em consideração a idade em anos completos na data de referência da Pnad.
\end{abstract}

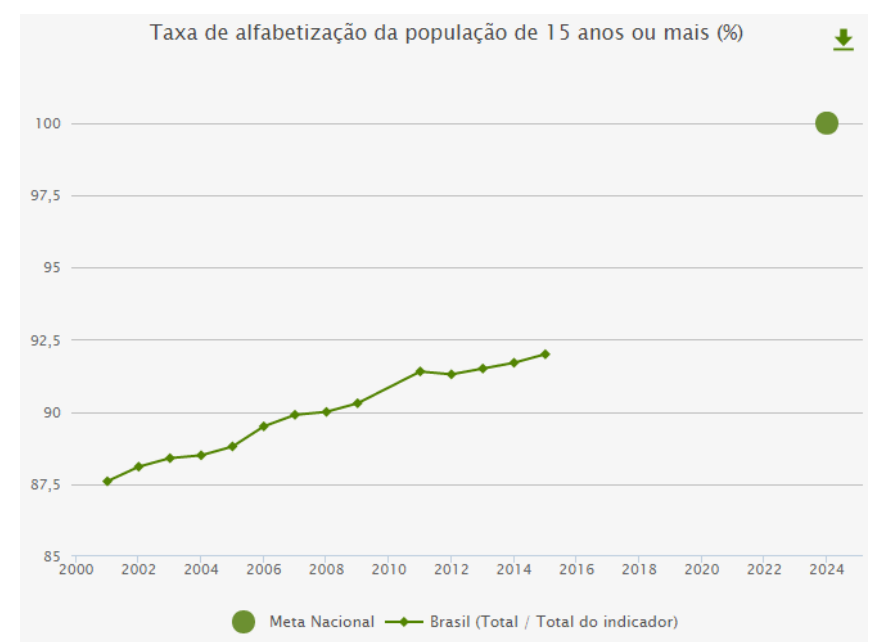

Fonte: Observatório do PNE (Plano Nacional de Educação)

O quadro abaixo apresenta dados para a taxa de alfabetização da população de 15 ou mais:

\begin{tabular}{|c|c|}
\hline Anos & $\begin{array}{c}\text { Porcentagem, na forma decimal, das pessoas } \\
\text { alfabetizadas de } \mathbf{1 5} \text { anos ou mais }\end{array}$ \\
\hline 2001 & 0,8760 \\
\hline 2002 & 0,8810 \\
\hline 2003 & 0,8840 \\
\hline 2004 & 0,8850 \\
\hline 2005 & 0,8880 \\
\hline 2006 & 0,8950 \\
\hline 2007 & 0,8990 \\
\hline 2008 & 0,9000 \\
\hline
\end{tabular}

8 Texto disponível em: http://www.observatoriodopne.org.br/metas-pne/9-alfabetizacao-educacaojovens-adultos/indicadores.

Criar Educação, Criciúma, v. 7, ำ1, jan/jul 2018.- PPGE - UNESC 


\begin{tabular}{|l|l|}
\hline 2009 & 0,9030 \\
\hline 2011 & 0,9140 \\
\hline 2012 & 0,9130 \\
\hline 2013 & 0,9150 \\
\hline 2014 & 0,9170 \\
\hline 2015 & 0,9200 \\
\hline
\end{tabular}

Fonte: Observatório do PNE (Plano Nacional de Educação)

Fonte: organizado a partir de Observatório do PNE

De posse de tais informações, os alunos definiram o seguinte problema: tendo em vista o crescimento do índice de alfabetização da população de 15 anos ou mais no país será possível em 2024 o Brasil alcançar a meta nacional de 100\% estipulado pelo Plano Nacional de Educação (PNE)?

Em busca de resposta a esse questionamento, decidiram que o trabalho seria desenvolvido em torno das variáveis tempo (em anos) e porcentagem (na forma decimal) de pessoas alfabetizadas maiores de 15 anos ou mais, e assumida a relação de dependência entre elas, de modo que a determinação da porcentagem de pessoas alfabetizadas de 15 anos ou mais ocorresse em função do tempo. Além disso, consideraram:

- $\quad x$ : tempo (em anos);

- $y$ : porcentagem de pessoas alfabetizadas de 15 anos ou mais (na forma decimal).

$\mathrm{Na}$ fonte consultada para a obtenção dos dados os alunos não conseguiram identificar a porcentagem de pessoas alfabetizadas de 15 anos ou mais referente ao ano de 2010 e justificativas para ausência desse dado. Tendo isso em vista e com base na figura e nos dados, assumiram as seguintes hipóteses:

- a porcentagem de pessoas alfabetizadas de 15 anos ou mais depende do tempo;

- a porcentagem de pessoas alfabetizadas de 15 anos ou mais desde 2001 até 2015 cresce linearmente, considerando a tendência da maioria dos dados, e esse comportamento permanecerá até 2024;

Criar Educação, Criciúma, v. 7, no1, jan/jul 2018.- PPGE - UNESC 
- como a porcentagem de pessoas alfabetizadas maiores de 15 anos ou mais desde 2001 até 2015 cresce linearmente, a ausência de valor para o ano de 2010 não interferirá no comportamento dos dados.

Com base nessas hipóteses, chegaram a uma conclusão de que o modelo matemático que possibilitaria a determinação da porcentagem de pessoas alfabetizadas de 15 anos ou mais em função do tempo seria um modelo da forma $y=a x+b$.

Diante desse modelo geral o trabalho foi encaminhado para a determinação dos valores de $a$ e $b$, de modo a tornar o modelo específico para o tema em questão. No entanto, como há algumas possibilidades de retas que podem se aproximar dos pontos indicados no quadro 2 , e tendo em vista outros trabalhos realizados na disciplina, em outros momentos de familiarização com tarefas de modelagem matemática, os alunos optaram pela utilização do método dos mínimos quadrados no caso linear para a determinação dos valores de $a$ e $b$. Com esse método, buscase o melhor ajuste dos pontos de modo a minimizar as diferenças entre a reta ajustada e os dados fornecidos.

Para isso, consideraram o seguinte sistema com duas equações e duas incógnitas:

$$
\left\{\begin{array}{l}
a \sum_{i=1}^{n} x_{i}^{2}+\mathrm{b} \sum_{i=1}^{n} x_{i}=\sum_{i=1}^{n} x_{i} y_{i} \\
a \sum_{i=1}^{n} x_{i}+\mathrm{bn}=\sum_{i=1}^{n} y_{i}
\end{array}\right.
$$

O processo de manipulação dos dados por esse método foi um pouco trabalhoso já que qualquer distração poderia ocasionar falhas posteriormente na ação de validação do mesmo. Assim sendo, os alunos decidiram então organizar o seguinte quadro:

Quadro 3: valores para o método dos mínimos quadrados

\begin{tabular}{|c|c|c|c|}
\hline$x_{i}$ & $y_{i}$ & $x_{i} \cdot y_{i}$ & $x_{i}^{2}$ \\
\hline 2001 & 0,8760 & 1752,876 & 4004001 \\
\hline 2002 & 0,8810 & 1763,762 & 4008004 \\
\hline
\end{tabular}

Criar Educação, Criciúma, v. 7, ํo1, jan/jul 2018.- PPGE - UNESC 


\begin{tabular}{|c|c|c|c|}
\hline 2003 & 0,8840 & 1770,652 & 4012009 \\
\hline 2004 & 0,8850 & 1773,540 & 4016016 \\
\hline 2005 & 0,8880 & 1780,440 & 4020025 \\
\hline 2006 & 0,8950 & 1795,370 & 4024036 \\
\hline 2007 & 0,8990 & 1804,293 & 4028049 \\
\hline 2008 & 0,9000 & 1807,200 & 4032064 \\
\hline 2009 & 0,9030 & 1814,127 & 4036081 \\
\hline 2011 & 0,9140 & 1838,054 & 4044121 \\
\hline 2012 & 0,9130 & 1836,956 & 4048144 \\
\hline 2013 & 0,9150 & 1841,895 & 4052169 \\
\hline 2014 & 0,9170 & 1846,838 & 4056196 \\
\hline 2015 & 0,9200 & 1853,800 & 4060225 \\
\hline & & & \\
\hline$\sum_{i=1}^{14} x_{i}=28110$ & $\sum_{i=1}^{14} y_{i}=12,5900$ & $\sum_{i=1}^{14} x_{i} \cdot y_{i}=25279,803$ & $\sum_{i=1}^{14} x_{i}^{2}=56441140$ \\
\hline
\end{tabular}

Fonte: os alunos

Considerando o sistema de equações apresentado anteriormente e os valores calculados conforme indicado no quadro 3, foi possível obter

$$
\left\{\begin{array}{l}
56441140 a+28110 b=25279,803 \\
28110 a+14 . b=12,5900
\end{array}\right.
$$

Ao resolver esse sistema, os alunos optaram por utilizar o método da adição. Decidiram multiplicar a equação $28110 . a+14 . b=12,5900$ por $-\frac{28110}{14}$. Desse modo, obtiveram:

$$
\left\{\begin{array}{l}
56441140 a+28110 b=25279,803 \\
28110 \cdot a \cdot\left(-\frac{28110}{14}\right)+14 \cdot b \cdot\left(-\frac{28110}{14}\right)=12,5900 \cdot\left(-\frac{28110}{14}\right)
\end{array}\right.
$$

Adicionando membro a membro as duas equações, determinaram a seguinte equação com uma incógnita:

$$
275,71 . a=0,88157
$$

Resolvendo essa equação, determinaram $a=0,003197454$, e logo após $b=-5,52074514$.

Retornando ao modelo geral $y=a x+b$ e fazendo as substituições necessárias, o modelo específico obtido foi $y=0,003197454 . x-5,52074514$.

Criar Educação, Criciúma, v. 7, ำ1, jan/jul 2018.- PPGE - UNESC 
Após a obtenção desse modelo matemático, os alunos iniciaram o processo de validação do mesmo. Para isso, construíram o seguinte quadro com o intuito de sintetizar as informações oriundas desse processo.

Quadro 4: Validação do modelo

\begin{tabular}{|c|c|c|c|}
\hline Ano & $\begin{array}{c}\text { Porcentagem } \\
\text { observada (na forma } \\
\text { decimal) }\end{array}$ & $\begin{array}{c}\text { Porcentagem } \\
\text { modelada (na forma } \\
\text { decimal) }\end{array}$ & $\begin{array}{c}\text { Diferença em relação ao } \\
\text { valor observado (na } \\
\text { forma decimal) }\end{array}$ \\
\hline 2001 & 0,8760 & 0,8774 & 0,0014 \\
\hline 2002 & 0,8810 & 0,8806 & $-0,0004$ \\
\hline 2003 & 0,8840 & 0,8838 & $-0,0002$ \\
\hline 2004 & 0,8850 & 0,8870 & 0,0020 \\
\hline 2005 & 0,8880 & 0,8902 & 0,0022 \\
\hline 2006 & 0,8950 & 0,8933 & $-0,0017$ \\
\hline 2007 & 0,8990 & 0,8965 & $-0,0025$ \\
\hline 2008 & 0,9000 & 0,8997 & $-0,0003$ \\
\hline 2009 & 0,9030 & 0,9029 & $-0,0001$ \\
\hline 2011 & 0,9140 & 0,9093 & $-0,0047$ \\
\hline 2012 & 0,9130 & 0,9125 & $-0,0005$ \\
\hline 2013 & 0,9150 & 0,9157 & 0,0007 \\
\hline 2014 & 0,9170 & 0,9189 & 0,0019 \\
\hline 2015 & 0,9200 & 0,9221 & 0,0021 \\
\hline
\end{tabular}

Fonte: os alunos

Considerando o modelo válido, tendo em vista que a diferença entre a quantidade observada e a quantidade modelada é pequena, como mostra a última coluna do quadro apresentado anteriormente, retornaram ao problema a fim de resolvê-lo. Substituindo $x$ por 2024 em $y=0,003197454 . x-5,52074514$ determinaram que $y=0,9509$. A partir isso, concluíram que para 2024 a meta de 100\% não será atingida, mas estará próximo desse objetivo.

\section{Considerações}

A intenção ao escrever esse artigo foi a de compartilhar uma experiência oriunda de um trabalho realizado em uma disciplina da licenciatura em Matemática em que os alunos após vivenciarem um processo de familiarização com tarefas de 
modelagem matemática foram responsáveis pela condução de todo o trabalho, ou seja, desde a escolha do tema até a resolução do problema.

Em relação a atividade desenvolvida vale salientar que foram necessárias algumas idas e vindas para sua realização. Foi um processo trabalhoso em relação à escolha de um tema e coleta de dados, além do próprio processo de obtenção do modelo, já que qualquer distração nessa ação, no que diz respeito a manipulação dos dados, poderia ocasionar falhas na ação de validação do mesmo. Cabe ressaltar também que é preciso se ter claro que as hipóteses definidas encaminharam para o modelo e resolução do problema aqui expostos e que a formulação de outras hipóteses poderiam conduzir a obtenção de outro modelo, com possivelmente outro resultado para o problema em tela, talvez até confirmando a previsão feita.

Em relação ao trabalho desenvolvido durante toda a disciplina acredita-se, primeiramente, que o contato com diferentes tarefas de modelagem matemática, ocorrido por diferentes momentos de inserção nas aulas, oportunizou aos futuros professores uma ideia de como poderão lidar com essa forma de condução da aula de Matemática com seus alunos e ter uma ideia da dinâmica dela, de papéis que podem ser assumidos pelo professor e pelos alunos durante a mesma. Em segundo, acredita-se também que a possibilidade de vivenciar a elaboração de uma modelagem matemática por completo oportunizou aos licenciandos ideias a respeito das dificuldades que seus alunos poderão ter no decorrer do trabalho com tarefas desse tipo e de intervenções que poderão fazer para auxiliá-los.

\section{Referências}

ALMEIDA, L. M. W.; DIAS, M. R. Um estudo sobre o uso da modelagem matemática como estratégia de ensino e aprendizagem. Bolema, Rio Claro, v. 17, n. 22, p. 1936, 2004.

ALMEIDA, L. M. W. Modelagem Matemática: um caminho para o pensamento reflexivo dos futuros professores de matemática. Contexto \& Educação, v. 1, p. 45$55,2006$.

BARBOSA, J. C. Integrando Modelagem Matemática nas práticas pedagógica.

Educação Matemática em Revista, São Paulo, v. 26, p. 17-25, 2009.

Criar Educação, Criciúma, v. 7, no1, jan/jul 2018.- PPGE - UNESC 
BASSANEZI, R. C. Ensino-aprendizagem com Modelagem Matemática. 3.ed. São Paulo: Contexto, 2011.

BRITO, D. S. Atribuição de Sentido e Construção de Significados em situações de Modelagem Matemática. 2004. Dissertação (Mestrado em Ensino de Ciências e Educação Matemática) - Universidade Estadual de Londrina, Londrina, 2004.

COSTA, M. L.; SILVA, K, A. P. Ideb do Ensino Médio: uma atividade de Modelagem Matemática. In: XII EPREM - Encontro Paranaense de Educação Matemática, 2014, Campo Mourão. Anais... Campo Mourão: UNESPAR, 2014.

FERREIRA, P. E. A. et al. Aquecimento Global: uma experiência com Modelagem Matemática. In: VI CNMEM - Conferência Nacional sobre Modelagem na Educação Matemática, 2009, Londrina. Anais...Londrina: UEL, 2009.

GEROLÔMO, A. M. L.; VERTUAN, R. E. Modelagem Matemática e o efeito estufa. In: VI CNMEM - Conferência Nacional sobre Modelagem na Educação Matemática, 2009, Londrina. Anais...Londrina: UEL, 2009.

OLIVEIRA, J. N.; SOTERO, B. S.; SILVA, K. A. P. Modelagem Matemática: Álcool e direção uma conscientização. In: XII EPREM - Encontro Paranaense de Educação Matemática, 2014, Campo Mourão. Anais... Campo Mourão: UNESPAR, 2014.

PARANÁ. Secretaria de Estado da Educação. Diretrizes Curriculares da Educação Básica: Matemática. Curitiba: SEED, 2008.

PEREIRA, L. L. et al. A Modelagem Matemática para o ensino da geometria relação de Euler. Criar Educação, Criciúma, v. 6, n.1, p. 01-12, 2017.

PFAHL, K. C. C. et al. Uma atividade de Modelagem Matemática desenvolvida: ritalina, usos e abusos. In: XII EPREM - Encontro Paranaense de Educação Matemática, 2014, Campo Mourão. Anais... Campo Mourão: UNESPAR, 2014.

SILVA, K. A. P.; VERTUAN, R. E. Decaimento radioativo: diferentes abordagens em uma atividade de Modelagem Matemática. In: X EPREM- Encontro Paranaense de Educação Matemática, 2009, Guarapuava. Anais...Guarapuava: UNICENTRO, 2009. 